\title{
Monitoring of Francisella noatunensis subsp. orientalis in farmed Nile tilapia (Oreochromis niloticus) in Brazil
}

\author{
Marianna Vaz Rodrigues ${ }^{1}$ - Claire Juliana Francisco ${ }^{2}$. \\ Gianmarco S. David ${ }^{3} \cdot$ Reinaldo José da Silva ${ }^{2}$. \\ Maria Fernanda Falcone-Dias ${ }^{3}$. \\ João Pessoa Araújo Júnior ${ }^{1}$
}

Received: 24 February 2017 / Accepted: 28 September 2017 / Published online: 7 October 2017

(C) Springer International Publishing AG 2017

\begin{abstract}
Francisella noatunensis orientalis is a bacterium that causes emerging bacteriosis in Nile tilapia (Oreochromis niloticus) in many parts of the world, including Brazil. It is a nonmotile, Gram-negative, strictly aerobic, facultative intracellular coccobacillus. This species of bacteria is responsible for low to high mortality in fish farms, causing economic losses for fish farmers. This study aimed to detect the presence of $F$. noatunensis orientalis using qPCR (realtime polymerase chain reaction) and to describe lesions caused by the bacterium in O. niloticus in Brazilian aquaculture. For this purpose, 360 fish from six fish farms (30 per farm) were sampled at two time points ( $n=180$ per sampling). Necropsies and histopathology were performed for lesion observation, in addition to qPCR and sequencing for detection and identification of Francisella species. Environmental data were collected using a multiparameter sonde YSI EXO2. All measured limnological variables were within the optimum range for cultivation of Nile tilapia. The major lesions present were melanization of the skin, splenomegaly, granulomas, and inflammatory cell responses. The prevalence of francisellosis varied from 0 to $86.66 \%$ between time periods and fish farms analyzed, and an outbreak was observed during the second sampling period. This study describes the prevalence of francisellosis in $O$. niloticus and reports that the lesions found are not exclusively associated with this bacterial disease.
\end{abstract}

These authors contributed equally to this work.

Marianna Vaz Rodrigues

mvazrodrigues@gmail.com

1 Department of Microbiology and Immunology, Biosciences Institute, Univ. Estadual Paulista (UNESP), Distrito de Rubião Júnior, Botucatu, SP 18618-689, Brazil

2 Department of Parasitology, Biosciences Institute, Univ. Estadual Paulista (UNESP), Distrito de Rubião Júnior, Botucatu, SP 18618-689, Brazil

3 São Paulo State Agency for Agribusiness Technology (APTA), CP 66 Jaú, Jaú, SP 17340-000, Brazil 
Keywords Francisellosis $\cdot$ Bacterial diseases $\cdot$ qPCR $\cdot$ Outbreak

\section{Introduction}

As the aquaculture industry intensifies and diversifies worldwide, it is natural that the domestication of new aquaculture species results in the recognition of "new" infectious agents and diseases, as has been repeatedly demonstrated over the years (Colquhoun and Duodu 2011). In recent years, bacteria of the genus Francisella have emerged as serious pathogens in various fish species, both farmed and wild, in various geographical regions worldwide (Kamaishi et al. 2005; Mauel et al. 2005; Hsieh et al. 2006; Nylund et al. 2006; Olsen et al. 2006; Birkbeck et al. 2011; Mauel et al. 2007).

Little information is available regarding if Francisella species are specific to which fish species. However, Francisella noatunensis orientalis is most commonly isolated from tilapia and causes disease in other fish species, including three-line grunt (Fukuda et al. 2002), a variety of ornamental cichlids (Hsieh et al. 2007), as well as in Gadus morhua (Atlantic cod), Salmo salar L. (Atlantic salmon), Parapristipoma trilineatum (three-line grunt), Morone chrysops $\times$ M. saxatilis (hybrid striped bass), and a shellfish species Haliotis gigantea (giant abalone) (Birkbeck et al. 2011). Additionally, experimental infections following intraperitoneal injection of $F$. noatunensis orientalis have been performed in red sea bream, Pagrus major (Kamaishi et al. 2010), and zebrafish, Danio rerio (Vojtech et al. 2009). Francisellosis primarily infects the fry and fingerling stages of fish, which exhibit systemic granulomatosis with multifocal granulomas in the internal organs, including the liver, spleen, and kidney (Soto et al. 2009; Soto et al. 2012).

Cases of francisellosis caused by $F$. noatunensis orientalis in farm-raised $O$. niloticus have been reported in Taiwan (Chen et al. 1994), Costa Rica (Soto et al. 2009), Indonesia (Ottem et al. 2009), the UK (Jeffery et al. 2010), and the USA (Soto et al. 2011). However, data regarding these bacterial infections at fish farms in South America are scarce. In Brazil, outbreaks of granulomatous disease in $O$. niloticus fingerlings reared in floating cages have been reported since 2012. Although the etiology of these cases is unclear, the clinical signs and field presentation of the disease were suggestive of francisellosis (Leal et al. 2014; Soto et al. 2014).

The aim of this study was to monitor the prevalence of $F$. noatunensis orientalis in Nile tilapia (O. niloticus) from Brazilian fish farms over a 5-month period using qPCR (real-time polymerase chain reaction) and to verify whether the lesions found could be associated with this species of bacteria.

\section{Materials and methods}

\section{Ethics statement}

This study was carried out in strict accordance with the following regulations: Law 11794/ 2008 and Decree 6899/2009. The protocol was approved by the Ethics Committee on Animal Use of the São Paulo State University (UNESP) (Protocol Number: 724-CEUA). The owners of fish farms used in this study consented to the use of their fish for detecting pathogens and for other analyses that are not included in this paper. 


\section{Sampling}

Oreochromis niloticus specimens were randomly sampled from cages of six fish farms from three different reservoirs in São Paulo State, Brazil, in November $2014(N=180)$ and March 2015 (180) (Table 1). Fish were sampled randomly (one/cage) until a total of 30 fish per farm were sampled.

Immediately after sampling, $O$. niloticus was put into a bottle to be euthanized by an overdose of MS-222 (1 g/L; Sigma-Aldrich). Euthanasia was achieved within 5 to $10 \mathrm{~min}$ according to fish body size. Then, all fishes were submitted to necropsy according to Noga (2010). In this step, all alterations were annotated in necropsy form.

The organs sampled for histopathology and molecular analyses were the brain, gall bladder, gill, gut, heart, kidney, liver, muscle, spleen, and stomach. Following the procedures described by Noga (2010), a $1-\mathrm{cm}^{3}$ portion of each tissue of all samples was fixed in $10 \%$ neutralbuffered formalin followed by processing using standard histological techniques and embedding in paraffin. Hematoxylin and eosin were used for staining.

\section{DNA extraction, qPCR (real-time polymerase chain reaction), and sequencing}

After sampling the tissues during necropsy in fish farms, these samples were frozen in $-20{ }^{\circ} \mathrm{C}$ and forwarded to the Molecular Diagnosis Laboratory of Microbiology and Immunology Department of Universidade Estadual Paulista (UNESP) for processing. Then, the organ tissues collected from each fish were pooled, and $20 \mathrm{mg}$ of each sample was used for molecular analysis. The DNA extraction was performed using the Wizard® SV Genomic DNA Purification System kit (Promega Corporation ${ }^{\circledR}$ ) according to the manufacturer's instructions. The DNA was eluted in elution buffer (nuclease-free water) and kept at $-20{ }^{\circ} \mathrm{C}$ until use. Purity and quantification of extracted DNA were measured using a 260/ 280 absorbance rate in a NanoDrop 2000c (Thermo Fisher Scientific@). Only DNA with a ratio of $>1.7(260 / 280)$ and with $>20 \mathrm{ng} / \mu \mathrm{L}$ was used in this study.

To quantify the presence of $F$. noatunensis subsp. orientalis in sampled fish, we performed qPCR using the Applied Biosystems 7500 Fast Real-Time PCR System (Applied Biosystems $\left.{ }^{\circledR}\right)$ with the SYBR Green method. The reaction mixture consisted of $12.5 \mu \mathrm{L}$ of GoTaq qPCR Master Mix 2× (Promega®), 10 pmol of each primer (iglC-forward and iglCreverse), $4 \mu \mathrm{L}$ of DNA, and nuclease-free water to adjust to $25 \mu \mathrm{L}$. As negative controls of the reaction, we used DNA from $O$. niloticus fresh from the laboratory and mix reaction without

Table 1 Number, weight $(\mathrm{g})$, and size $(\mathrm{cm})$ of Oreochromis niloticus sampled from six fish farms in the first and second sampling periods

\begin{tabular}{lllllll}
\hline Fish farm & Number & Weight $^{\mathrm{a}}(\bar{X})$ & Weight $^{\mathrm{b}}(\bar{X})$ & Size $^{\mathrm{a}}(\bar{X})$ & Size $^{\mathrm{b}}(\bar{X})$ & River basin \\
\hline A & 30 & 518.67 & 441.10 & 19.53 & 21.64 & Paranapanema \\
B & 30 & 434.76 & 518.99 & 22.33 & 22.00 & Tiete \\
C & 30 & 286.55 & 537.56 & 19.49 & 22.02 & Tiete \\
D & 30 & 427.47 & 430.86 & 21.42 & 20.73 & Tiete \\
E & 30 & 234.08 & 396.82 & 18.43 & 22.77 & Grande Paraná \\
F & 30 & 304.17 & 424.77 & 19.71 & 21.49 & Grande Paraná \\
Total & 180 & 367.61 & 458.35 & 20.15 & 21.77 & \\
\hline
\end{tabular}

\footnotetext{
${ }^{\text {a }}$ First sampling

${ }^{\mathrm{b}}$ Second sampling
} 
sample. To evaluate specificity of the reaction for Francisella, the following positives controls, Aeromonas hydrophila, Edwardsiella tarda, Mycobacterium spp., and tissue of Nile tilapia obtained healthy from the laboratory, were used and were not amplified.

The 88-bp amplicons were purified using illustra MicroSpin ${ }^{\mathrm{TM}} \mathrm{S}-400 \mathrm{HR}$ Columns (GE Healthcare $\left.{ }^{\circ}\right)$ according to the manufacturer's instructions for identification by Sanger sequencing. For this purpose, the purified amplicon was sequenced in both directions using the BigDye $^{\mathrm{TM}}$ Terminator Cycle Sequencing Kit (Applied Biosystems ${ }^{\circledR}$ ) on an Applied Biosystems capillary 3500 Genetic Analyzer. The quality of the electropherograms was assessed using Sequencing Analysis version 5.4 (Applied Biosystems). Finally, sequences were identified by the Basic Local Alignment Search Tool (BLAST) algorithm.

\section{Environmental data}

To evaluate whether the water quality affected the infection rate of pathogens, environmental parameters were analyzed. Data were collected using a multiparameter sonde YSI EXO2 equipped with sensors for measuring temperature, conductivity, dissolved oxygen, $\mathrm{pH}$, and total dissolved solids. Data were collected from surface water at a depth of $1 \mathrm{~m}$, where cages are located.

\section{Statistical analysis}

The prevalence of $F$. noatunensis orientalis as detected by qPCR was calculated for each fish farm sampled. The occurrence of observed lesions and of positive qPCR results was also analyzed. All statistical analyses were performed using Statistica v. 10 (StatSoft 2011) and visualized using GraphPad Prism version 5.00 for Windows (GraphPad Software, San Diego, California, USA, www.graphpad.com).

\section{Results}

\section{Macroscopic and microscopic lesions}

In all fish farms evaluated in this study, producers had a daily record of mortality, which is $30 \%$. During necropsy, we observed many lesions common in bacterial infections, including hemorrhage, skin ulcers, and melanization of the skin (Table 2). The prevalence varied between fish farms (Figs. 3 and 4), but no significant difference was observed between the two time periods analyzed. Although this paper reports the occurrence and describes lesions in $O$. niloticus caused by Francisella, we observed that other bacteria were present in samples and that this microorganism was negative, with the following prevalence of 3-26\% of A. hydrophila, 10-26,66\% of E. tarda, and 13-56\% of Mycobacterium sp.

The microscopic lesions are shown in Figs. 1 and 2. The most prevalent lesions were the presence of inflammatory cells, bacteria, and granulomas. The spleen and kidney contained higher quantities of granulomas, and more granulomas were observed during the second sampling period.

\section{Molecular results}

Amplification using the primers iglC-forward and iglC-reverse (Soto et al. 2010) only detected F. noatunensis orientalis. Genomic and unspecific signals were not observed. After 


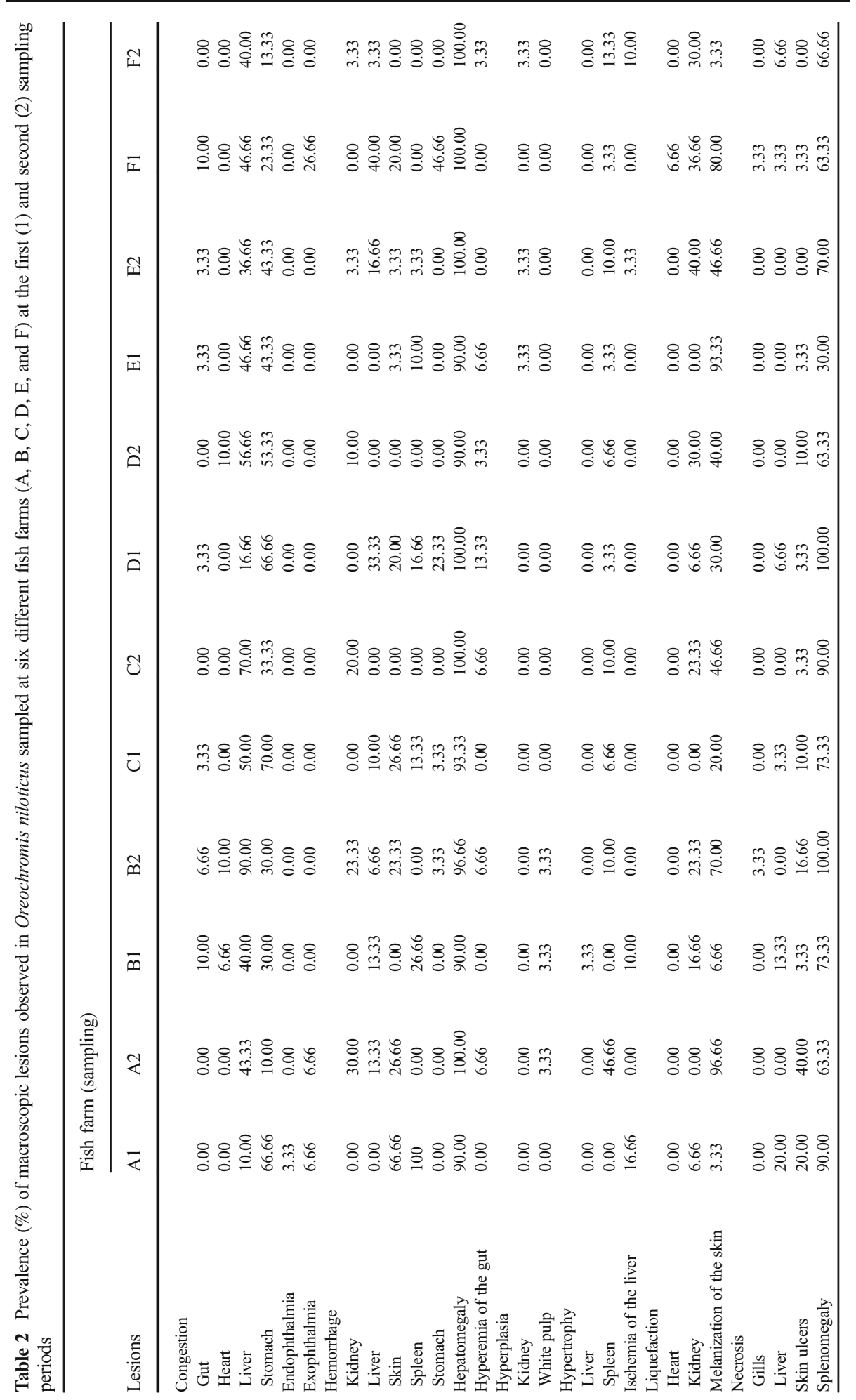



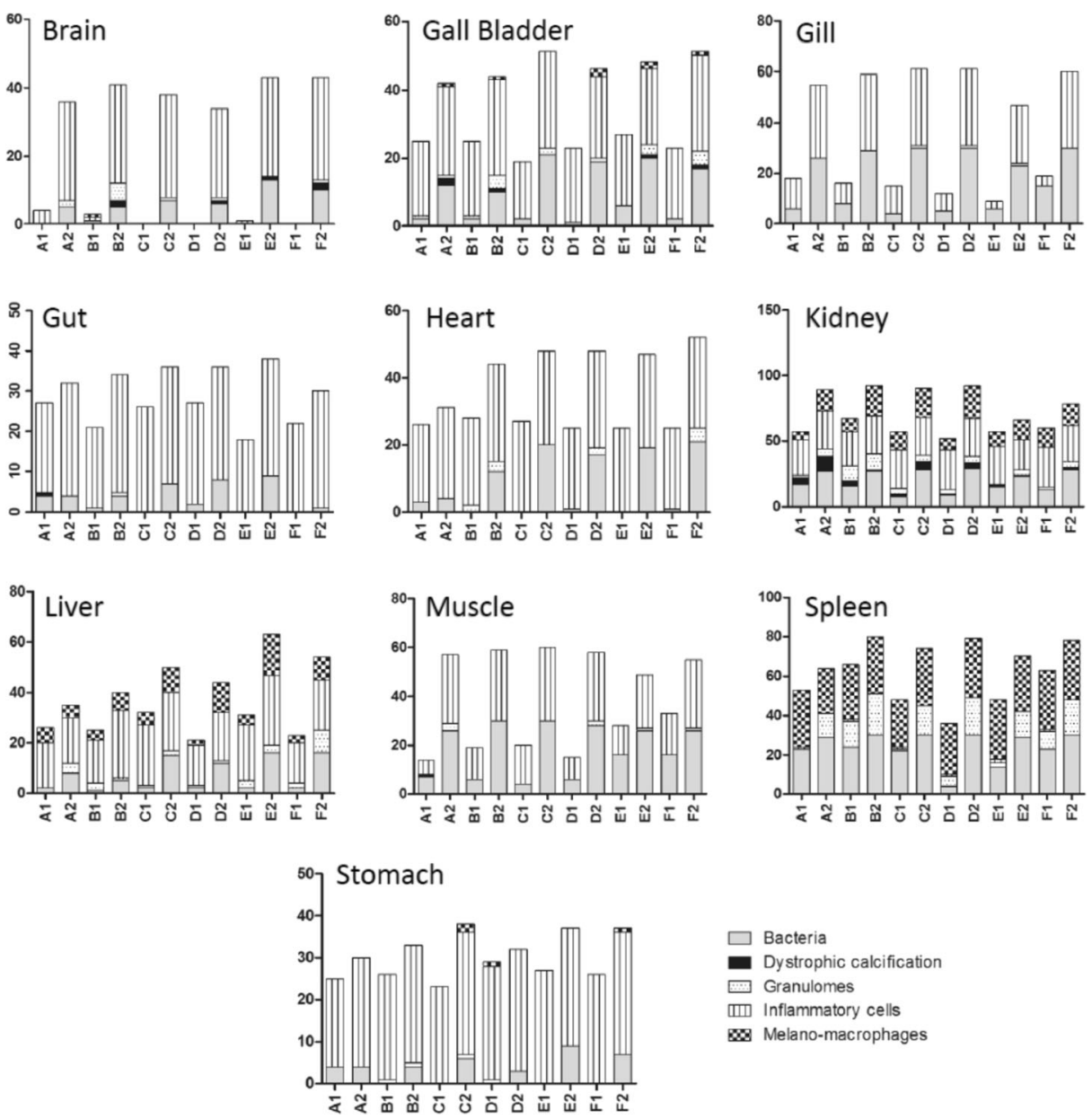

Fig. 1 Prevalence of microscopic lesions observed in O. niloticus sampled from six fish farms (A, B, C, D, E, and F) at two time points (1 and 2)

sequencing, the amplicons indicated $100 \%$ similarity to $F$. noatunensis orientalis (GenBank: CPO12153), confirming the presence of francisellosis in these samples (Figs. 3 and 4).

Analyzing Ct values (supplementary material), in the first sampling, sample 11 had the lowest $\mathrm{Ct}$ value (26.36) and 7 and 29 the highest level (34.74), both from fish farm B. In the second sampling, $\mathrm{Ct}$ values vary from 30.44 to 36.79 , with fish farm $\mathrm{D}$ having the lowest $\mathrm{Ct}$ value and fish farm A having the highest level. An increase in the detection of this bacterium was observed in the second sampling at all fish farms analyzed (Figs. 3 and 4), indicating an outbreak of francisellosis.

\section{Environmental data}

All environmental variables studied (Table 3) were within the range favorable for growing tilapia as identified by Webster and Lim (2006). Most variables indicated a slight decrease in water quality in the second sampling period, when a severe drought affected southeastern 


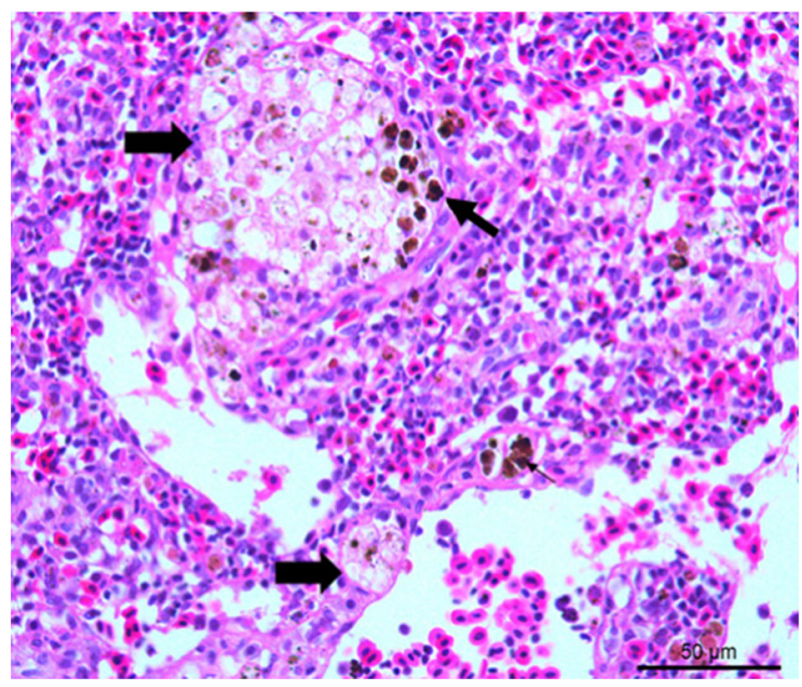

Fig. 2 Hematoxylin and eosin staining of splenic lesions caused by bacteria. Presence of granulomas (thick arrow) and melano-macrophages (thin arrow)

Brazil and affected the level and water exchange rate of all reservoirs. The $\mathrm{pH}$ ranged from 7.1 to 9.2, with a small decrease in the second sampling period at all fish farms. The conductivity and the total dissolved solids showed the opposite pattern, increasing in the second sampling period. The temperature varied from 25.6 to $31.3{ }^{\circ} \mathrm{C}$, which is within the optimum range for culturing Nile tilapia. Dissolved oxygen was less than $5 \mathrm{mg}$ per liter in fish farms B, C, and F in the second sampling period.

\section{Discussion}

Francisella noatunensis is an important and emerging pathogen responsible for outbreaks of francisellosis in fish farms in numerous countries (Leal et al. 2014). Natural infections of F. noatunensis orientalis in O. niloticus have been associated with a $95 \%$ mortality rate (Chern

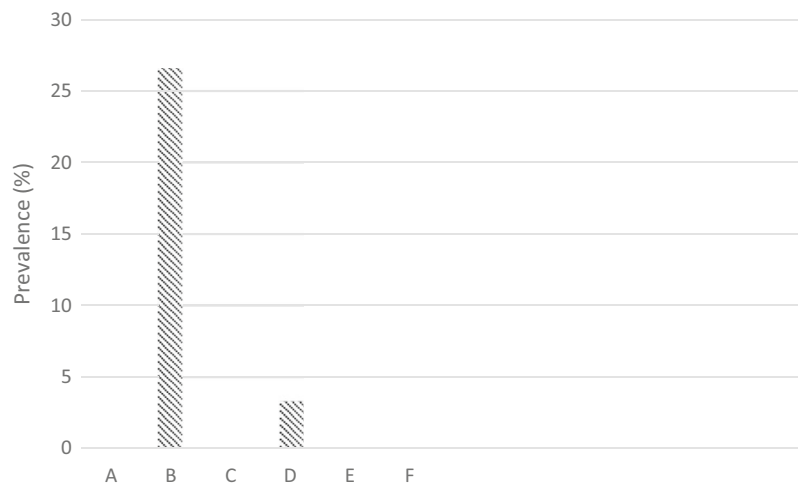

Fig. 3 Prevalence (\%) of Francisella noatunensis orientalis based on qPCR in Oreochromis niloticus sampled at six different fish farms (A, B, C, D, and F) in the first sampling period 


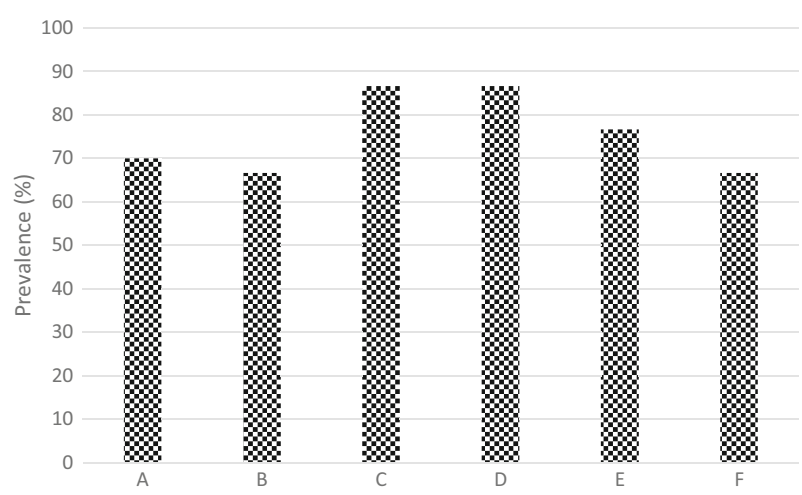

Fig. 4 Prevalence (\%) of Francisella noatunensis orientalis based on qPCR in Oreochromis niloticus sampled at six different fish farms (A, B, C, D, and F) in the second sampling period

and Chao 1994), which is much higher than the mortality rates observed in this study, which recorded a mortality rate of approximately $30-40 \%$ at each of the fish farms analyzed. Comparable to this study, Leal et al. (2014) observed mortality rates of 30-60\% in an outbreak of francisellosis in $O$. niloticus and reported that the mortality rate could be determined by environmental parameters, such as water temperature. These authors described an increase in mortality when the water reached temperatures below $22{ }^{\circ} \mathrm{C}$ and no mortality when temperatures were higher than $24{ }^{\circ} \mathrm{C}$. As reported by these authors, the conditions in the floating cages (mainly high stock densities and intensive husbandry) of the farms in Brazil, combined with water temperature variations, could suppress the immune responses in fish. This observation may explain the high prevalence of disease observed in the second sampling period in this research (Figs. 3 and 4), during which a severe drought occurred, resulting in changes in all water parameters, thereby affecting the immune system of the fish and ultimately causing disease.

Table 3 Water environmental data monitoring at six different fish farms (A, B, C, D, E, and F) at the first (1) and second (2) sampling periods

\begin{tabular}{llllll}
\hline $\begin{array}{l}\text { Fish } \\
\text { farm }\end{array}$ & First sampling & \multicolumn{5}{l}{} \\
\cline { 2 - 6 } & $\mathrm{pH}$ & Temp. $\left({ }^{\circ} \mathrm{C}\right)$ & $\begin{array}{l}\text { Dissolved oxygen } \\
(\mathrm{mg} / \mathrm{L})\end{array}$ & $\begin{array}{l}\text { Conductivity }(\mu \mathrm{S} / \\
\mathrm{cm})\end{array}$ & $\begin{array}{l}\text { Total dissolved solids } \\
(\mathrm{mg} / \mathrm{L})\end{array}$ \\
& $\mathrm{M} \pm \mathrm{Sd}$ & $\mathrm{M} \pm \mathrm{Sd}$ & $\mathrm{M} \pm \mathrm{Sd}$ & $\mathrm{Sd}$ & $\mathrm{M} \pm \mathrm{Sd}$ \\
\hline $\mathrm{A}$ & $8.18 \pm 0.01$ & $26.87 \pm 0.00$ & $8.20 \pm 0.01$ & $191.70 \pm 0.14$ & $125.0 \pm 0.00$ \\
$\mathrm{~B}$ & $8.37 \pm 0.09$ & $26.08 \pm 0.00$ & $8.25 \pm 0.01$ & $179.60 \pm 0.00$ & $117.00 \pm 0.00$ \\
$\mathrm{C}$ & $9.19 \pm 0.00$ & $27.75 \pm 0.01$ & $8.25 \pm 0.00$ & $184.43 \pm 0.06$ & $120.00 \pm 0.00$ \\
$\mathrm{D}$ & $9.12 \pm 0.00$ & $27.81 \pm 0.02$ & $8.46 \pm 0.01$ & $52.82 \pm 0.00$ & $34.00 \pm 0.00$ \\
$\mathrm{E}$ & $7.49 \pm 0.01$ & $28.52 \pm 0.00$ & $6.64 \pm 0.01$ & $64.13 \pm 0.05$ & $42.00 \pm 0.00$ \\
$\mathrm{~F}$ & $7.39 \pm 0.01$ & $31.36 \pm 0.01$ & $6.16 \pm 0.01$ & $64.50 \pm 0.00$ & $42.00 \pm 0.00$ \\
Second & sampling & & & $252.50 \pm 0.00$ & $164.00 \pm 0.00$ \\
$\mathrm{~A}$ & $7.71 \pm 0.00$ & $27.09 \pm 0.00$ & $9.14 \pm 0.01$ & $220.10 \pm 0.00$ & $143.00 \pm 0.00$ \\
$\mathrm{~B}$ & $8.10 \pm 0.00$ & $28.30 \pm 0.00$ & $4.56 \pm 0.01$ & $232.60 \pm 0.00$ & $151.00 \pm 0.00$ \\
$\mathrm{C}$ & $7.32 \pm 0.00$ & $28.58 \pm 0.00$ & $4.32 \pm 0.00$ & $107.10 \pm 0.00$ & $70.00 \pm 0.00$ \\
$\mathrm{D}$ & $7.44 \pm 0.00$ & $29.06 \pm 0.00$ & $5.20 \pm 0.00$ & $77.60 \pm 0.00$ & $50.00 \pm 0.00$ \\
E & $7.46 \pm 0.00$ & $29.58 \pm 0.00$ & $7.11 \pm 0.00$ & &
\end{tabular}


Environmental conditions, particularly temperature and the density of each fish cage, appear to play a significant role in the mortality rate. The higher the temperature, the more acute the disease is likely to be, with mortality levels increasing with temperature until a maximum pathogenic temperature is reached (Kamaishi et al. 2010; Ostland et al. 2006). This finding is well reported in the study of Duodu and Colquhoun (2010), which observed that the culturability of the tested strains decreased with increasing water temperature. The presence of mixed infections including other pathogenic bacteria may also significantly affect mortality rates (Colquhoun and Duodu 2011), and such mixed infections were also detected in the fish sampled (data not presented in this paper). Although the principal reservoirs of Francisella spp. remain unknown, transmission of francisellosis in fish has an obvious connection with the aquatic environment, and this group of bacteria has been isolated from both marine and freshwater environments (Duodu et al. 2012). Presently, there is limited knowledge regarding the ecology of Francisella spp. in aquatic environments (Duodu and Colquhoun 2010). Moreover, the worsening of water quality can lead to decreased fish immunity, thereby making them more susceptible to infection, which may be one explanation for the outbreak that occurred in the second sampling period of this study.

As shown in Figs. 3 and 4, in the first sampling period, F. noatunensis orientalis was not detected at some fish farms, whereas this bacterium was detected at all fish farms in the second sampling period. This observation may result from the difference in the water quality parameters, as mentioned before, or from the spread of the microorganism due to the capacity of the water to transmit francisellosis.

All described incidences of francisellosis in fish manifest as systemic, chronic, granulomatous infections resulting in varying degrees of mortality. Common observations of francisellosis in fish, like $O$. niloticus, include the extensive occurrence of white, partly protruding nodules (granuloma) of varying size in the spleen, kidney, and liver (Kamaishi et al. 2010; Olsen et al. 2006; Ostland et al. 2006; Hsieh et al. 2007). Such nodules were not observed in this study, suggesting that if the animals did not present these signs, they could be negative for this bacterium. Other organs may be affected, as associated pathological changes have also been described in the gill, heart, testes, musculature, brain, and eye (Camus et al. 2013). These pathological changes were also observed in this research but with lower occurrence. In this study, nodules or granulomas were not observed during necropsy, only in histopathology; therefore, we suggest that histopathology is crucial for observing these types of lesions. Additionally, the absence of nodules or granulomas in necropsy did not exclude the possibility of infection of Francisella spp. in fish. Kamaishi et al. (2010) also reported no granulomas associated with the first report of francisellosis in a non-vertebrate, i.e., Haliotis spp.

Olsen et al. (2006) described in G. morhua infected with francisellosis the enlargement of the spleen and reported an extensive chronic granulomatous inflammation with multiple granulomas in all organs in histopathological findings, with few to numerous bacteria sometimes observed within granuloma. Our results also showed splenomegaly (Table 2) as the most prevalent lesion in the $O$. niloticus analyzed, suggesting an infection process. Also observed in this study (Figs. 1 and 2) were the kidney and spleen being the organs with the highest quantity of granuloma-containing bacteria. Other signs may be present in infected fish, such as emaciation, dark coloration, raised hemorrhagic nodules, and skin ulcerations (Mauel et al. 2005), all of which were observed at various prevalence rates in this study (Table 2).

The major clinical signs of francisellosis observed by Leal et al. (2014) were melanosis, exophthalmia, erratic swimming, gill pallor, nephromegaly, skin ulcers (primarily at the base of the fin), splenomegaly, and hepatomegaly, all of which were accompanied by multifocal white 
nodules, in fingerlings, juveniles, and young adults. In this study, we also observed melanosis, skin ulcers, splenomegaly, and hepatomegaly, but without nodules, indicating that the observation of macroscopic lesions, which had no nodules or granulomas, should not be the only technique used for diagnosing francisellosis in fish.

In the histopathological analysis, infected fish could present extensive granulomatous inflammation which are dominated by hypertrophied foamed macrophages (Olsen et al. 2006; Mauel et al. 2007). Mauel et al. (2005) described focal to diffuse necrosis and necrotizing vasculitis in affected organs, accompanied by infiltration of mononuclear cells and granuloma formations. Few or no bacteria may be observable, particularly in cases of advanced disease with extensive mature granulomas (Nylund et al. 2006). Such lesions may be observed in almost any organ or tissue type, including meninges in severe infections (Mauel et al. 2007), explaining why this study found bacteria, inflammatory cells, and granuloma in all tissues analyzed (Fig. 1). This research found the same lesions as described by Mauel et al. (2007), but the spleen and kidney were the organs with the highest quantity of granulomas, while the spleen and muscle were the organs with the highest prevalence of bacteria colonies. Birkbeck et al. (2011) and Colquhoun and Duodu (2011) also mention that lesions were found most frequently in the spleen and kidney and explained that these organs are the most severely affected, although lesions have been described in all organ systems. Notably, the increase in the quantity of bacteria and lesions caused by bacteria observed in the second sampling period of this study suggests an outbreak of bacteriosis, in this case, francisellosis.

Other bacteria that affect fish can result in the same alterations in necropsy and histology as previously described. We observed that some samples with granulomas, inflammatory cells, and bacteria in histopathology were negative for $F$. noatunensis orientalis, indicating that the presence of these alterations does not necessarily imply francisellosis. Therefore, it is important to perform qPCR to confirm the etiology, as is done for the diagnosis of bacteria such as Aeromonas, Edwardsiella, Mycobacterium, and others that cause similar lesions.

Due to the economic losses caused by mortality and disease development, monitoring and surveillance are crucial for controlling bacteriosis. Therefore, many researchers report the utility of molecular techniques, especially real-time PCR because of its sensitivity, specificity, and rapidity (Soto et al. 2009; Soto et al. 2010; Soto et al. 2013). Therefore, we used qPCR to detect F. noatunensis orientalis in this study. Additionally, Soto et al. (2010) validated the reaction performed in this study using other bacteria that can cause granuloma, including Mycobacterium marinum, Nocardia spp., Streptococcus agalactiae, S. iniae, Edwardsiella ictaluri, E. tarda, Aeromonas spp., Vibrio spp., and Flavobacterium columnare; the reaction only amplified F. noatunensis orientalis, indicating its specificity. For all these reasons, the detection by realtime PCR and identification by sequencing used in this research are very trustworthy.

Soto et al. (2013) considered molecular diagnosis to be the appropriate technique for the detection and surveillance of pathogens. Moreover, molecular diagnostics are being evaluated for use in monitoring for the presence of this bacterium in cultured and wild O. niloticus on and around the island of Oahu, a technique that could also be applied in other countries. This information is very important for many countries such as Brazil that do not have a surveillance program for francisellosis. As shown in this study, depending on the environment, handling, and fish immunity, a lack of surveillance can lead to high disease prevalence, which can lead to mortality and economic losses to the producers.

According to Leal et al. (2014), outbreaks of granulomatous disease in O. niloticus fingerlings reared in floating cages in Brazil have been reported since 2012. These authors also reported that although the etiology of these cases was unclear, the clinical signs and field 
presentation of the disease were suggestive of francisellosis. Until now, there were no reports of this bacteriosis in adult fish in Brazil. Therefore, the mortality in our study was likely lower than that found in previous studies due to the immunity of the fish and the fact that previous research focused on different taxa. Additionally, this study observed that the lesions described could be caused by bacterial infections other than francisellosis, indicating that there is no association between this type of alteration and francisellosis. Therefore, it is important to investigate the etiology of all infections using other techniques with high specificity and sensitivity, such as qPCR.

Although Francisella tularensis and F. philomiragia have zoonotic potential, particularly in individuals with suppressed immunity, F. noatunensis orientalis does not present a risk to humans (Colquhoun and Duodu 2011). Therefore, the primary risk associated with francisellosis is the economic loss resulting from the zootechnical performance index and mortality.

\section{Conclusions}

This work found a prevalence in the first sampling from 3 to $26 \%$, and in the second sampling from 66 to $86 \%$. It was also shown that the absence of macroscopic granulomas does not mean that the fish is negative for francisellosis. Therefore, it is important to use additional tools for investigation and surveillance of this disease, such as histopathology and molecular tests.

Acknowledgements The authors thank FAPESP for the financial support (2013/50504-5) and postdoctoral fellowships (2014/15859-0 to C.J. Francisco, 2014/13718-0 to M.V. Rodrigues, and 2015/13025-7 to M.F. Falcone-Dias). The authors also thank CNPq for the doctoral fellowship to R.J. da Silva of CNPq (307808/20149) and CNPq-PROTAX (440496/2015-2)/FAPESP 2016/50377-1.

Compliance with ethical standards All procedures performed in studies involving animals were in accordance with the ethical standards of the institution or practice at which the studies were conducted.

Conflict of interest The authors declare that they have no conflict of interests.

\section{References}

Birkbeck TH, Feist SW, Verner-Jeffreys DW (2011) Francisella infections in fish and shellfish. J Fish Dis 34:173-187 Camus AC, Dill JA, McDermott AJ et al (2013) Francisella noatunensis subsp. orientalis infection in IndoPacific reef fish entering the United States through the ornamental fish trade. J Fish Dis 36:681-684

Chen SC, Tung MC, Chen SP et al (1994) Systematic granulomas caused by a rickettsia-like organism in Nile tilapia, Oreochronuis niloticus (L.), from southern Taiwan. J Fish Dis 17:591-599

Chern RS, Chao CB (1994) Outbreaks of a disease caused by rickettsia-like organism in cultured tilapias in Taiwan. Fish Pathol 29:61-71

Colquhoun DJ, Duodu S (2011) Francisella infections in farmed and wild aquatic organisms. Vet Res 42(1):1-15

Duodu S, Colquhoun D (2010) Monitoring the survival of fish-pathogenic Francisella in water microcosms. FEMS Microbial Ecol 74:534-541

Duodu S, Larsson P, Sjödin A et al (2012) The distribution of Francisella-like bacteria associated with coastal waters in Norway. Microbial. Ecol 64(2):370-377

Fukuda Y, Okamura A, Nishiyama M et al (2002) Granulomatosis of cultured three-line grunt Parapristipoma trilineatum caused by an intracellular bacterium. Fish Pathol 37:119-124

Hsieh CY, Tung MC, Tu C et al (2006) Enzootics of visceral granulomas associated with Francisella-like organism infection in tilapia (Oreochromis spp.) Aquaculture 254:129-138 
Hsieh C, ZB W, Tung MC et al (2007) PCR and in situ hybridization for the detection and localization of a new pathogen Francisella-like bacterium (FLB) in ornamental cichlids. Dis Aquat Org 75:29-36

Jeffery KR, Stone D, Feist SW et al (2010) An outbreak of disease caused by Francisella sp. in Nile tilapia Oreochromis niloticus at a recirculation fish farm in the UK. Dis Aquat Org 91:161-165

Kamaishi T, Fukuda Y, Nishiyama M (2005) Identification and pathogenicity of intracellular Francisella bacterium in three-line grunt Parapristipoma trilineatum. Fish Pathol 4:67-71

Kamaishi T, Miwa S, Goto E et al (2010) Mass mortality of giant abalone Haliotis gigantea caused by a Francisella sp. bacterium. Dis Aquat Org 89:145-154

Leal CAG, Tavares GC, Figueiredo HCP (2014) Outbreaks and genetic diversity of Francisella noatunensis subsp orientalis isolated from farm-raised Nile tilapia (Oreochromis niloticus) in Brazil. Genet Mol Res 13(3):5704-5712

Mauel MJ, Miller DL, Styer E et al (2005) Occurrence of Piscirickettsiosis-like syndrome in tilapia in the continental United States. J Vet Diagn Investig 17:601-605

Mauel MJ, Soto E, Moralis JA (2007) A piscirickettsiosis-like syndrome in cultured Nile tilapia in Latin America with Francisella spp. as the pathogenic agent. J Aquat Anim Health 9:27-34

Noga EJ (2010) Fish diseases: diagnosis and treatment. Blackwell Publishing, Iowa

Nylund A, Ottem KF, Watanabe K et al (2006) Francisella sp. (Family Francisellaceae) causing mortality in Norwegian cod (Gadus morhua) farming. Arch Microbiol 185:383-392

Olsen AB, Mikalsen J, Rode M et al (2006) A novel systemic granulomatous inflammatory disease in farmed Atlantic cod, Gadus morhua L., associated with a bacterium belonging to the genus Francisella. J Fish Dis 29:307-311

Ostland VE, Stannard JA, Creek JJ et al (2006) Aquatic Francisella-like bacterium associated with mortality of intensively cultured hybrid striped bass Morone chrysops x M. saxatilis. Dis Aquat Org 72:135-145

Ottem KF, Nylund A, Karlsbakk E et al (2009) Elevation of Francisella philomiragia subsp. noatunensis Mikalsen et al. (2007) to Francisella noatunensis comb. nov. [syn. Francisella piscicida Ottem et al. (2008) syn. nov.] and characterization of Francisella noatunensis subsp. orientalis subsp. nov., two important fish pathogens. J Appl Microbiol 106:1231-1243

Soto E, Hawke JP, Fernandez D et al (2009) Francisella sp., an emerging pathogen of tilapia, Oreochromis niloticus (L.), in Costa Rica. J Fish Dis 32:713-722

Soto E, Bowles K, Fernandez D et al (2010) Development of a real-time PCR assay for identification and quantification of the fish pathogen Francisella noatunensis subsp. orientalis. Dis Aquat Org 89:199-207

Soto E, Baumgartner W, Wiles J et al (2011) Francisella asiatica as the causative agent of piscine francisellosis in cultured tilapia (Oreochromis sp.) in the United States. J Vet Diagn Investig 23:821-825

Soto E, Illanes O, Hilchie D et al (2012) Molecular and immunohistochemical diagnosis of Francisella noatunensis subsp. orientalis from formalin-fixed, paraffin-embedded tissues. J Vet Diagn Investig 24: $840-845$

Soto E, McGovern-Hopkins K, Klinger-Bowen R et al (2013) Prevalence of Francisella noatunensis subsp. orientalis in cultured tilapia on the island of Oahu, Hawaii. J Aquat Anim Health 25:104-109

Soto E, Primus AE, Pouder DB et al (2014) Identification of Francisella noatunensis in novel host species French grunt (Haemulon Flavolineatum) and Caesar grunt (Haemulon carbonarium). J Zoo Wildl Med 45(3):727-731

StatSoft, Inc (2011) STATISTICA (data analysis software system), version 10. http://www.statsoft.com. Cited 10 May 2016

Vojtech LN, Sanders GE, Conway C et al (2009) Host immune response and acute disease in a zebrafish model of Francisella pathogenesis. Infect Immun 77:914-925

Webster CD, Lim C (2006) Tilapia: biology, culture, and nutrition. CRC Press 